\title{
Análisis factorial confirmatorio y propiedades psicométricas de la Escala de Desesperanza de Beck en estudiantes en contextos de pobreza en México
}

\author{
Francisco Quiñonez-Tapia, Tanya E. Méndez Luévano y Nicté Castañeda-Camey \\ Universidad de Guadalajara, México
}

\begin{abstract}
Confirmatory factor analysis and psychometric properties of the Beck Hopelessness Scale in students in contexts of poverty in Mexico. Hopelessness predicts depression and suicide. In the world, suicide is the second cause of death in people between 15 and 29 years, and depression is the main cause of disability. The internal factorial structure, reliability and construct validity of the Hopelessness Scale were analyzed in upper secondary and higher education students in contexts of poverty in Mexico. 771 participants were evaluated. Reliability was examined with the coefficient omega, and validity with confirmatory factor analysis with the weighted least square mean and variance adjusted method. The value of omega was .89 . The confirmatory factor analysis revealed an SRMR $=.07$, CFI $=.95, \mathrm{TLI}=.94, \mathrm{RMSEA}=.05$. The scale is valid and reliable for students in contexts of poverty in México.
\end{abstract}

Keywords: Reliability; validity; students; Beck Hopelessness Scale.

Resumen: La desesperanza predice la depresión y el suicidio. En el mundo, el suicidio es la segunda causa de muerte en personas de 15 a 29 años, y la depresión es la principal causa de incapacidad. Se analizó la estructura factorial interna, fiabilidad y validez de constructo de la Escala de Desesperanza de Beck en estudiantes de educación media superior y superior en contextos de pobreza en México. 771 participantes fueron evaluados. Se examinó la fiabilidad con el coeficiente omega, y la validez con el análisis factorial confirmatorio con el método weighted least square mean and variance adjusted. El valor de omega fue de .89. El análisis factorial confirmatorio arrojó un $\mathrm{SRMR}=.07, \mathrm{CFI}=.95, \mathrm{TLI}=.94, \mathrm{RMSEA}=.05$. La escala es válida y fiable para los estudiantes en contextos de pobreza en México.

Palabras clave: Fiabilidad; validez; estudiantes; Escala de Desesperanza de Beck.

\section{Introducción}

La desesperanza incluye un conjunto de estructuras cognitivas interrelacionadas y constituidas por las expectativas negativas sobre la vida que determinan una perspectiva desalentadora del futuro (Beck, Weissman, Lester, y Trexler, 1974). Además, la desesperanza predice el desarrollo de la depresión (Horwitz, Berona,

Recibido: 23 de marzo 2019: aceptado: 10 de julio 2019

Correspondencia: Francisco Quiñonez Tapia, Universidad de Guadalajara, Centro Universitario de Ciencias Económico-Administrativas, Periférico Norte No. 799, Núcleo Universitario los Belenes, C.P 45100, Zapopan, Jalisco, México. Correo-e: fcoqtapia@yahoo.com.mx
Czyz, Yeguez, y King, 2017) y el suicidio (Burr, Rahm-Knigge, y Conner, 2018; Lane y Miranda, 2018; Pérez, Marco, y García-Alandete, 2017; Sánchez-Teruel et al., 2018; Silva et al., 2017). El suicidio es la segunda causa de muerte en el mundo entre las personas de 15 a 29 años; el 78\% de los suicidios ocurren en países de ingresos económicos bajos y medianos. Así mismo, la depresión es la principal causa de incapacidad a nivel mundial (Organización Mundial de la Salud [OMS], 2017).

El suicidio en México se incrementó del 2007 al 2017 en un $49 \%$ de casos por año; de 4.395 suicidios se pasó a 6.559 respectivamente (Instituto Nacional de Estadística y Geografía [INEGI], 2017a). Además, el 
$30.5 \%$ (33.381.777) de la población mexicana informó haberse sentido deprimida en algún momento (INEGI, 2017b). Por lo tanto, la intervención oportuna sobre la desesperanza es importante para prevenir el desarrollo de conductas de depresión y suicidio.

Beck et al. (1974) construyeron la Escala de Desesperanza de Beck (BHS) con el propósito de identificar el nivel de desesperanza en pacientes con conducta suicida. La BHS tiene tres factores correlacionados: Afectivo, Conductual y Cognitivo. Para Beck et al. el factor de Sentimientos sobre el Futuro aborda los sentimientos de felicidad, entusiasmo, esperanza y fe que tiene el sujeto con respecto su futuro; el factor de Falta de Motivación se refiere a las posibilidades que tiene un sujeto de presentar una conducta de renuncia a todo acto que le permita desarrollarse en un futuro; y el factor de Expectativas sobre el Futuro se refiere a todos aquellos pensamientos que un sujeto elabora con respecto a su vida en el futuro.

La fiabilidad y la validez de la BHS han sido cuestionadas por más de 40 años. Como resultado, se han referido diversas estructuras factoriales: un factor, en sujetos con depresión (Young, Halper, Clark, Scheftner, y Fawcett, 1992), y en estudiantes de primaria y secundaria (Kagotho, Bowen, Ssewamala, Vaughn, y Kirkbride, 2018); dos factores relacionados, en población con esquizofrenia (Kao, Liu, y Lu, 2012), depresión (Dyce, 1996), cáncer (Nissim et al., 2010; Spangenberg et al., 2016), cuidadores de pacientes psiquiátricos (Aloba, Ajao, Alimi, y Esan, 2016), y en población general (Satorres et al., 2016); tres factores, en participantes con bipolaridad, esquizofrenia, depresión, intento suicidio (Aloba et al., 2015), ansiedad (Steer, Beck, y Brown, 1997), abuso de sustancias (Steer, Iguchi, y Platt, 1994), y con población general (Iliceto y Fino, 2015; Kocalevent et al., 2016).

Asimismo, se han encontrado soluciones factoriales válidas de cuatro factores en participantes con conducta suicida (Rueda-Jaimes et al., 2016; Zhang, Jia, Hu, Qiu, y Liu, 2015) y puérperas (Gonzales-Huerta et al., 2013), y cinco factores con población general (Zhang et al., 2015), con poblaciones clínicas no psiquiátricas (Innamorati et al., 2014; Szabó et al., 2016), y con población general (Kliem, Lohmann, Mößle, y Brähler, 2018). Otros estudios han validado la BHS con población de estudiantes universitarios (Aloba, Awe, Adelola, y Aloba, 2018, Boduszek y Dhingrana, 2016), Chang, D'Zurilla, y Maydeu, 1994; Hanna, 2011; Steed, 2001; Pompili, Tatarelli, Rogeres, y Lester, 2007; González, 2009).

Los resultados de Iliceto y Fino (2015) con población general y universitaria coinciden con los resultados de Beck et al. (1974). Igualmente, el estudio de Nissim et al. (2010), en población con cáncer, arrojó una estructu- ra de dos factores correlacionados conformados por los reactivos positivos y negativos respectivamente, como fue propuesto inicialmente por Beck et al. En México, solo se dispone del estudio de Córdova y Rosales (2011), quienes evaluaron las propiedades psicométricas de la BHS en estudiantes de nivel medio superior, y concluyeron una fiabilidad aceptable y una estructura factorial inaceptable, y sugirieron elaborar una nueva escala. Partiendo de la heterogeneidad de resultados relacionados con la estructura de la BHS, mediante la presente investigación se ha pretendido llevar a cabo un estudio riguroso estadísticamente con objeto de tratar de clarificar tanto la estructura factorial como las propiedades psicométricas de la BHS, basándonos en una muestra en estudiantes de educación media-superior y superior en contextos de pobreza de México.

\section{Método}

\section{Participantes}

Se evaluaron a 771 estudiantes de educación media superior y superior del norte de Jalisco en México. El sistema nacional de educación de México tiene la siguiente estructura: (a) Educación básica, incluye prescolar con tres años de duración y atiende a población entre 3 a 6 años de edad, primaria con duración de 6 años con población de 6 a 12 años de edad y secundaria con tres años de duración con estudiantes de los 12 a 15 años de edad; (b) Educación media superior con tres años de estudio y atiende a estudiantes con edad entre 15 a 18 años, y existen dos tipos de bachillerato: general y tecnológico, y (c) Educación superior, que incluye licenciatura con programas de formación entre 4 a 5 años en promedio y con población con un rango entre 18 a 23 años de edad y posgrado (maestría y doctorado). Por otro lado, se eligió la región norte de Jalisco porque es representativa de la dinámica de pobreza en México. En este sentido, el 80\% (64.205) de los habitantes del norte de Jalisco experimenta pobreza, mientras que, en la nación, el 51.2\% (62.717.200) de la población vive con pobreza (Consejo Nacional de Evaluación de la Política de Desarrollo Social [CONEVAL], 2016).

El tamaño de la muestra se determinó con la fórmula de Gabaldón (1980) para poblaciones finitas, y el resultado fue un total de 385 participantes, de un universo de 8.223 estudiantes de educación media y superior en el norte de Jalisco (Secretaría de Educación Jalisco, 2017). Posteriormente se duplicó la muestra para evitar el error Tipo I para lograr que los análisis factoriales fueran estables y generalizables (Koran, 2016; Morata-Ramírez, Holgado-Tello, Barbero-García, y Méndez, 2015). 
Además, se realizó un muestreo aleatorio simple y se identificó a los estudiantes con las listas de asistencia. Aplicamos los siguientes criterios de inclusión: (a) estudiantes de educación media y superior del norte de Jalisco, (b) para el caso de los adultos, participar voluntariamente en el estudio y expresarlo con el consentimiento informado, y (c) para el caso de los adolescentes, participar voluntariamente en el estudio y con autorización del responsable legal, expresados ambos a través del consentimiento informado. Por otro lado, los criterios de exclusión establecidos fueron (a) abandonar el estudio y (b) no encontrarse en la institución en el momento de la aplicación. Las sedes de la aplicación fueron en las distintas instituciones de educación al norte de Jalisco.

El rango de edad de los participantes fue de 15 a 44 años $\left(M_{\text {edad }}=20, D T=4\right)$, y la media del puntaje total de la BHS fue de $3.5(D T=2.9)$. La Figura 1 muestra el número de participantes en cada fase del estudio. Los catorce estudiantes que no aceptaron participar fueron menores de edad debido a que los tutores legales no firmaron el consentimiento informado. Los dos participantes que abandonaron el estudio mencionaron no estar interesados después de revisar el instrumento. Los estudiantes no reclutados fueron porque no se encontraron en las instituciones al momento de la aplicación.

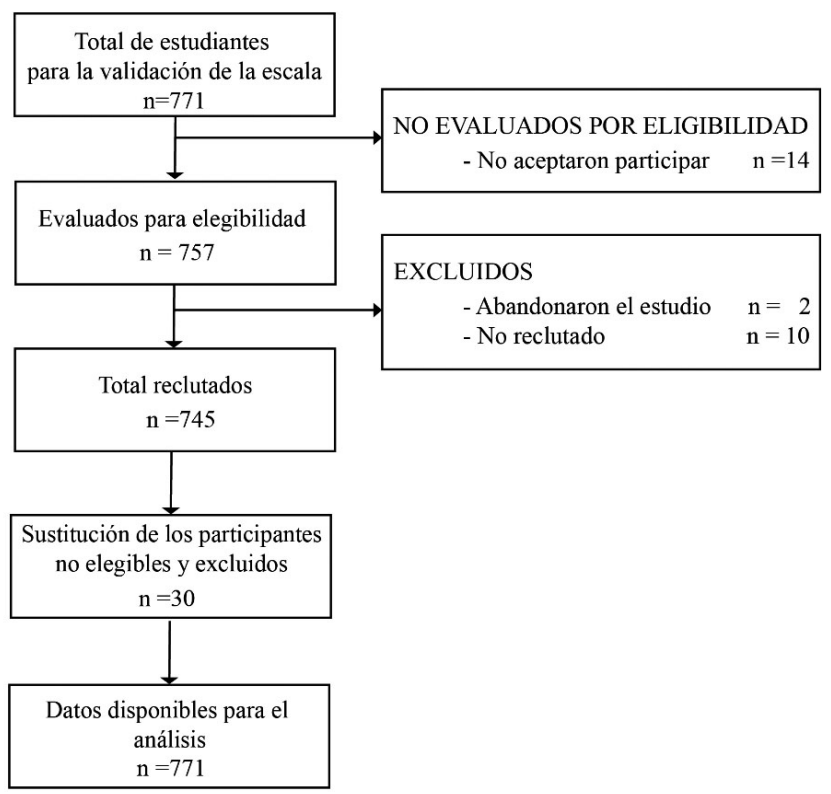

Figura 1. Descripción del número de participantes en cada fase del estudio.

\section{Instrumentos}

Escala de Desesperanza de Beck [Beck Hopelessness Scale] (BHS; Beck et al., 1974; versión en español de
Aguilar et al., 1995). La escala de desesperanza consta de 20 reactivos de respuesta dicotómica (verdadero o falso) con un rango de puntuación de 0 a 20 , y distribuidos en tres factores: factor de Sentimientos sobre el Futuro (afectivo: reactivos 1, 6, 13, 15 y 19); el factor de Falta de Motivación (conductual: reactivos 2, 3, 9, 11, 12, 16, 17 y 20); y Expectativas sobre el Futuro (cognitivos: reactivos 4, 7, 8, 14 y 18). (Véase el Anexo I).

Inventario de Depresión de Beck (BDI; Beck et al., 1961; versión en español de Beltrán, Freyre, y Hernández-Guzmán, 2012). El BDI consta de 20 reactivos de cuatro opciones de respuesta con un rango de puntuación de 0 a 63 . Es un inventario de autoinforme de síntomas depresivos experimentados en la semana previa a la aplicación. La BDI ha sido validada para población mexicana por Beltrán et al. con adolescentes $(\alpha=.92)$, y por Jurado et al. (1998) con población general $(\alpha=.87)$. Asimismo, se obtuvo un valor de omega de McDonald de .96 para la población del presente estudio.

Inventario de Ansiedad de Beck (BAI; Beck et al., (1988); versión en español de Vázquez et al., 2015). El BAI tiene 21 reactivos de respuesta tipo Likert que va de 0 (nada) a 3 (severo) con un rango de puntuación total de 0 a 63, y es un instrumento de autoinforme de ansiedad generalizada. LA BAI ha sido validada en población mexicana por Vázquez et al., (2015) con población con cáncer $(\alpha=.92)$. En el presente estudio se encontró un valor de omega de McDonald de .97 .

\section{Procedimiento}

Primero, se presentó el proyecto a los directores de las instituciones educativas y se solicitó el permiso preceptivo. Se explicó a los estudiantes el estudio y se les invitó a participar. Por un lado, los estudiantes adultos expresaron su voluntad de participar en el estudio a través del consentimiento informado y se procedió a la aplicación de los instrumentos. Por otra parte, a los adolescentes que aceptaron participar en el estudio se les entregó una hoja de presentación y contacto del equipo de investigación y el consentimiento informado por escrito para ser firmado por el estudiante y el tutor legal, y se les citó al día siguiente para llevar a cabo la aplicación de los instrumentos. Los participantes no obtuvieron ningún tipo de beneficio por su participación. Finalmente, los instrumentos de evaluación fueron respondidos por los estudiantes en una sola sesión con un promedio máximo de 20 minutos.

Se atendieron a los criterios éticos establecidos en el reglamento de la ley general de salud en materia de investigación para la salud (1983) en México, en el código ético del psicólogo en México (Sociedad Mexicana de 
Psicología [SMP], 2010), en la Asociación Americana de Psicología (APA, 2017) y a lo establecido en la declaración Helsinki (World Medical Association [WMA], (2017). De esta manera, se consideró el estudio sin riesgo para la salud, y se garantizó la participación voluntaria y la confidencialidad de los sujetos del estudio. Por último, la investigación fue aprobada por un comité de ética de la Universidad de Guadalajara.

\section{Análisis estadístico}

Se evaluó la fiabilidad de la BHS con el coeficiente omega de McDonald y se estableció el valor de omega igual o superior a .70 para aceptar la escala como fiable. Se determinó la validez factorial de la BHS con el análisis factorial confirmatorio (AFC) para distintos modelos (Véase Tabla 2.), y se utilizó el método de weighted least square mean and variance adjusted (WLMSV) debido a que permite analizar la matriz de correlaciones tetracóri- cas para datos categóricos binarios (Flora y Curran, 2004; Liang y Yang, 2014).

Asimismo, se estimaron los índices de ajuste: chi-cuadrado $\left(\chi^{2}\right)$, standardized root mean square residual (SRMR), comparative fit index (CFI), Turker-Lewis index (TLI), root mean square error of approximation (RMSEA) y Akaike information criterion (AIC). Se realizó un análisis factorial multigrupo para valorar la invarianza de la BHS en los grupos por sexo, estadios del desarrollo humano, y nivel educativo. De acuerdo con Hu y Bentler (1999), se establecieron los siguientes valores de bondad de ajuste: RMSEA igual o menor a .06 (bueno), CFI y TLI $\geq .90$ (aceptable), y TLI y CFI $\geq$ .95 (bueno) para valorar el ajuste de los datos a los modelos. El valor de AIC se calculó para comparar entre los modelos; el valor más bajo indica el mejor modelo (Huang, 2017).

Se analizó la validez concurrente a partir de correlacionar los resultados de la BHS con el BDI y el BAI. También,

Tabla 1. Puntuaciones medias y DTs de la Escala de Desesperanza (BHS) según características sociodemográficas

\begin{tabular}{|c|c|c|c|c|c|c|}
\hline \multirow{2}{*}{ Variable } & \multirow{2}{*}{$n$} & \multirow{2}{*}{$\%$} & \multirow{2}{*}{ Media } & \multirow{2}{*}{$D T$} & \multicolumn{2}{|c|}{ ANOVA } \\
\hline & & & & & $F$ & $p$ \\
\hline Sexo & & & & & 1.32 & .25 \\
\hline Hombres & 338 & 44 & 3.36 & 2.68 & & \\
\hline Mujeres & 433 & 56 & 3.62 & 3.10 & & \\
\hline Edad (años) & & & & & .92 & .46 \\
\hline 15 a 17 & 244 & 31 & 3.39 & 3.03 & & \\
\hline 18 a 22 & 406 & 53 & 3.53 & 2.82 & & \\
\hline 23 a 27 & 85 & 11 & 6.65 & 3.31 & & \\
\hline 28 a 32 & 24 & 3 & 3.83 & 2.18 & & \\
\hline 33 a 37 & 4 & 1 & 5.75 & 3.86 & & \\
\hline 38 a 44 & 8 & 1 & 3.83 & 3.86 & & \\
\hline Estadios del desarrollo humano ${ }^{a}$ & & & & & 1.01 & .31 \\
\hline Adolescencia & 410 & 53 & 3.33 & 2.86 & & \\
\hline Edad Adulta & 361 & 47 & 3.69 & 3.69 & & \\
\hline Nivel educativo & & & & & 5.68 & .02 \\
\hline Educación media superior & 331 & 43 & 3.19 & 2.89 & & \\
\hline Educación superior & 440 & 57 & 3.74 & 2.94 & & \\
\hline Ingreso familiar mensual & & & & & 2.35 & .07 \\
\hline Menos de $\$ 2,599$ (USD\$ 128) & 297 & 39 & 3.74 & 3.00 & & \\
\hline A $\$ 3,800(\mathrm{USD} \$ 150)$ & 247 & 32 & 3.54 & 2.98 & & \\
\hline A $\$ 5,000(\mathrm{USD} \$ 246)$ & 118 & 15 & 3.16 & 2.67 & & \\
\hline Más de $\$ 5,000$ (USD\$246) & 109 & 14 & 3.16 & 2.86 & & \\
\hline
\end{tabular}

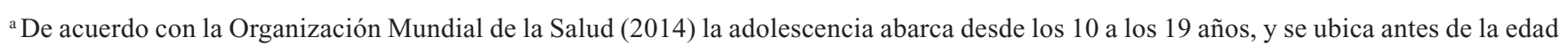
adulta. 
se determinó la validez predictiva con la regresión lineal de la desesperanza como variable independiente y la depresión y ansiedad como variables dependientes. Por otro lado, se determinó la validez discriminante de la BHS con el análisis de las curvas ROC (receiver operating characteristic).

Por otra parte, se partió del supuesto de que el dato ausente fue al azar y se trató mediante el método de imputación múltiple. Además, se procesaron los datos con el software Mplus 8.1 para determinar el coeficiente omega de McDonald y la validez factorial, y con el software SPSS 25 para determinar la validez concurrente, predictiva discriminante, la tabla de frecuencias de los datos sociodemográficos y la prueba de ANOVA.

\section{Resultados}

En la Tabla 1 se indican las frecuencias y porcentajes de los participantes por sexo, edad, estadios del desarrollo humano, nivel educativo e ingreso familiar. Asimismo, se describen las medias, desviaciones típicas y los resultados de la prueba de ANOVA para las puntuaciones totales obtenidas en la BHS por variables sociodemográficas. Se aprecia que solo existen diferencias significativas asociadas a los niveles educativos.

\section{Análisis factorial confirmatorio}

La Tabla 2 muestra los resultados del AFC de los modelos evaluados. Los modelos presentaron valores de $\chi^{2}$ elevados y significativos $(p<.05)$, lo que indica que la matriz de los datos observados difiere de la matriz esperada. Esto es muy común para muestras superiores a 200 casos, por lo que se sugiere analizar otros índices de ajuste (Bentler y Bonett, 1980). Los índices de ajuste aplicados a los modelos de la tabla arrojaron que los datos de la investigación tuvieron mejores ajustes a los modelos propuestos y probados empíricamente por Beck et al. (1974) con los veinte reactivos: dos factores correlacionados (negativos y positivos), y tres factores correlacionados. Asimismo, se demuestra que el modelo de Beck et al. de segundo orden tiene índices que indican un buen ajuste. Por lo tanto, el factor de Desesperanza es de segundo orden que se expresa a partir de los factores de Expectativa sobre el Futuro, Falta de Motivación y Sentimientos sobre el futuro como lo plantearon teóricamente Beck et al., aunque sin probarlo empíricamente.

Los valores de AIC para los modelos propuestos por Beck et al (1974) indicaron que el modelo de tres factores correlacionados fue el de mejor ajuste. Sin embargo, los valores de AIC del modelo de dos factores correlacionados y del modelo de segundo orden (ambos de Beck et al., 1974) son cercanos. Por lo tanto, se elige el modelo pro- puesto de Beck et al. de segundo orden, toda vez que los datos de la investigación se ajustan al modelo, responde a la teoría y prueba empíricamente el modelo (ver Figura 2).

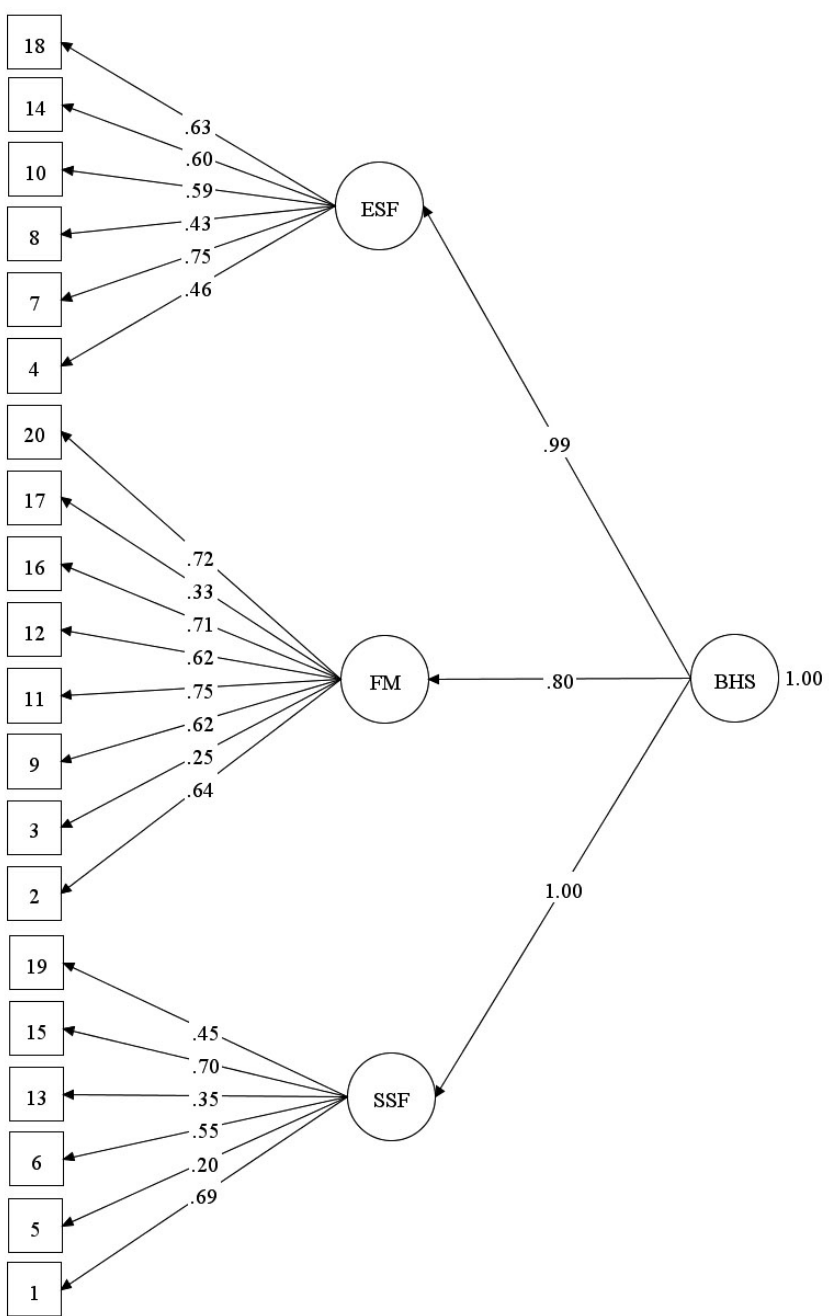

Figura 2. Solución final del análisis factorial confirmatorio del modelo de Beck et al. (1974 de segundo orden. SSF = Sentimientos sobre el Futuro, FM = Falta de Motivación, ESF = Expectativas sobre el Futuro; BHS = Escala de Desesperanza.

\section{Invarianza}

Se evaluó la invarianza del modelo de Beck et al. (1974) de segundo orden para verificar si la estructura del modelo estaba presente en cada grupo por sexo, estadios del desarrollo humano y nivel de estudio. En la Tabla 3 se pueden observar los resultados del análisis factorial confirmatorio por grupos separados y el análisis factorial confirmatorio multigrupo. El análisis factorial confirmatorio por grupos separados muestra una ligera invarianza entre los grupos por sexo. Sin embargo, los análisis factoriales multigrupo demuestran que no 
Tabla 2. Índices de ajuste para los diferentes modelos de análisis factorial confirmatorio de la BHS

\begin{tabular}{|c|c|c|c|c|c|c|c|c|}
\hline \multicolumn{2}{|r|}{ Modelo } & \multicolumn{7}{|c|}{ Índices de ajuste } \\
\hline Autor & Estructura factorial & $\chi^{2}$ & $g l$ & SRMR & CFI & TLI & RMSEA $[90 \% \mathrm{CI}]$ & AIC \\
\hline \multicolumn{9}{|l|}{ Un factor } \\
\hline Chang et al., $1994^{\mathrm{a}}$ & (F1) $1-20$ & $297 *$ & 170 & .08 & .93 & .92 & $.03[.02, .04]$ & 12286 \\
\hline Hanna et al., 2011 a & (F1) $6,7,9,15$ & $0.5^{*}$ & 2 & .01 & 1.00 & 1.00 & $.00[.00, .05]$ & 2733 \\
\hline \multicolumn{9}{|c|}{ Dos factores correlacionados } \\
\hline Beck et al., 1974 & $\begin{array}{l}\text { (F1) } 2,4,7,9,11,12,14,16,17,18,20 \\
\text { (F2) } 1,3,5,6,8,10,13,15,19\end{array}$ & $256^{*}$ & 169 & .07 & .95 & .95 & $.03[.02, .03]$ & 12241 \\
\hline Beck et al., 1974: SF & $\begin{array}{l}\text { (F1) } 2,4,7,9,11,12,14,16,17,18,20 \\
\text { (F2) } 1,3,6,8,13,15,19\end{array}$ & $400 *$ & 136 & .10 & .83 & .81 & $.05[.05, .06]$ & 11481 \\
\hline Steed et al., 1974 & $\begin{array}{l}\text { (F1) } 1,3,6,7,10,11,15,18,19 \\
\text { (F2) } 2,9,12,14,16,17,20\end{array}$ & $173^{*}$ & 103 & .07 & .95 & .94 & $.03[.02, .03]$ & 9956 \\
\hline Pompilini, 2007a & $\begin{array}{l}\text { (F1) } 11,16,17,19,20 \\
\text { (F2) } 1,7,12,14,15,18\end{array}$ & $64^{*}$ & 43 & .06 & .96 & .95 & $.03[.02, .03]$ & 6779 \\
\hline Aloba et al., 2018 a & $\begin{array}{l}\text { (F1) } 11,12,13,14,15,16,17,18,19,20 \\
\text { (F2) 4,6,7,8,9,10 }\end{array}$ & $303 *$ & 105 & .10 & .85 & .93 & $.05[.04, .06]$ & 9957 \\
\hline \multicolumn{9}{|c|}{ Tres factores correlacionados } \\
\hline Beck et al., 1974 & $\begin{array}{l}\text { (F1) } 1,5,6,13,15,19 \\
\text { (F2) } 2,3,9,11,12,16,17,20 \\
\text { (F3) } 4,7,8,10,14,18\end{array}$ & $248^{*}$ & 167 & .07 & .96 & .95 & $.03[.02, .03]$ & 12238 \\
\hline Beck et al., 1974: SF & $\begin{array}{l}\text { (F1) } 1,6,13,15,19 \\
\text { (F2) } 2,3,9,11,12,16,17,20 \\
\text { (F3) } 4,7,8,14,18\end{array}$ & $640^{*}$ & 135 & .14 & .68 & .64 & $.07[.06, .08]$ & 11483 \\
\hline \multicolumn{9}{|c|}{ Cinco factores correlacionados } \\
\hline González, 2009ª & $\begin{array}{l}\text { (F1) } 1,15,3,10,13,8 \\
\text { (F2) } 12,11,18,16 \\
\text { (F3) } 4,14,9,2 \\
\text { (F4) } 17,19,20 \\
\text { (F5) } 6,5\end{array}$ & $611^{*}$ & 147 & .14 & .71 & .67 & $.06[.06, .07]$ & 11928 \\
\hline \multicolumn{9}{|l|}{ Segundo orden } \\
\hline Beck et al., 1974 & $\begin{array}{l}\text { (F1) } 1,6,13,15,19,5 \\
\text { (F2) } 2,3,9,11,12,16,17,20 \\
\text { (F3) } 4,7,8,14,18,10\end{array}$ & $262 *$ & 168 & .07 & .95 & .94 & $.03[.02, .03]$ & 12251 \\
\hline Beck et al., 1974: SF & $\begin{array}{l}\text { (F1) } 1,6,13,15,19 \\
\text { (F2) } 2,3,9,11,12,16,17,20 \\
\text { (F3) } 4,7,8,14,18\end{array}$ & $230 *$ & 132 & .07 & .94 & .93 & $.03[.02, .04]$ & 11571 \\
\hline \multicolumn{9}{|l|}{ Multirasgo-multimétodo } \\
\hline & $\begin{array}{l}\text { Método 1: (Negativos) } \\
\text { 2,4,7,9,11,12,14,16,17,18,20 } \\
\text { Método 2: (Positivos) } \\
1,3,5,6,8,10,13,15,19\end{array}$ & $872 *$ & 150 & .16 & .60 & .50 & $.08[.07, .08]$ & 12382 \\
\hline $\begin{array}{l}\text { Boduszek y Dhingra, } \\
2018^{\mathrm{a}}\end{array}$ & $\begin{array}{l}\text { (F1) } 1,5,6,13,15,19 \\
\text { (F2) } 2,3,9,11,12,16,17,20 \\
\text { (F3) } 4,7,8,10,14,18\end{array}$ & & & & & & & \\
\hline
\end{tabular}

Nota: chi cuadrado $(\chi 2)$; grados de libertad (g1); standardized root mean square residual (SRMR); comparative fit index (CFI), Turker-Lewis index (TLI), root mean square error of approximation (RMSEA), Akaike information criterion (AIC). SF: Solución final sin el reactivo cinco y diez.

${ }^{a}$ Estudios realizados con estudiantes universitarios.

$* p<.05$. 
existe invarianza de la estructura factorial del modelo de Beck et al. (1974) de segundo orden dentro de los grupos de análisis. Por otro lado, se observa que de acuerdo con el análisis único del SRMR los valores presentados para los distintos grupos están por encima de un valor de .80 , lo que hace rechazar el ajuste de los modelos. Sin embargo, un análisis integral de los demás índices de ajuste permite aceptar los modelos. Por lo tanto, el modelo de segundo orden de Beck et al. (1974) presenta una estructura factorial equivalente dentro de los grupos por sexo, estadios del desarrollo humano y nivel de estudios.

Tabla 3. Índices de ajuste para los modelos de invarianza

\begin{tabular}{|c|c|c|c|c|c|c|}
\hline Grupos/Invarianza $^{\mathrm{a}}$ & $\chi^{2}$ & $g l$ & $S R M R$ & CFI & TLI & RMSEA [IC 90\%] \\
\hline \multicolumn{7}{|l|}{ Análisis factorial confirmatorio } \\
\hline \multicolumn{7}{|l|}{ Sexo } \\
\hline Hombres & $235^{*}$ & 169 & .12 & .88 & .86 & $.03[.02, .04]$ \\
\hline Mujeres & $215^{*}$ & 169 & .08 & .96 & .96 & $.02[.01, .04]$ \\
\hline \multicolumn{7}{|l|}{ Estadios del desarrollo humano ${ }^{\mathrm{b}}$} \\
\hline Adolescencia & $231^{*}$ & 169 & .10 & .93 & .92 & $.02[.02, .04]$ \\
\hline Adultos & $232 *$ & 169 & .10 & .92 & .91 & $.03[.02, .04]$ \\
\hline \multicolumn{7}{|l|}{ Nivel educativo } \\
\hline Educación media superior & $211^{*}$ & 169 & .11 & .94 & .93 & $.03[.01, .04]$ \\
\hline Educación superior & $255^{*}$ & 169 & .10 & .91 & .90 & $.03[.03, .04]$ \\
\hline \multicolumn{7}{|c|}{ Análisis factorial confirmatorio multigrupo } \\
\hline \multicolumn{7}{|l|}{ Sexo } \\
\hline Configuracional & $433^{*}$ & 340 & .10 & .95 & .94 & $.03[.02, .04]$ \\
\hline Escalar & $445^{*}$ & 351 & .10 & .95 & .94 & $.03[.02, .04]$ \\
\hline \multicolumn{7}{|l|}{ Estadios del desarrollo humano ${ }^{\mathrm{b}}$} \\
\hline Configuracional & $433 *$ & 340 & .10 & .95 & .94 & $.03[.02, .04]$ \\
\hline Escalar & $445^{*}$ & 351 & .10 & .95 & .94 & $.03[.02, .04]$ \\
\hline \multicolumn{7}{|l|}{ Nivel Educativo } \\
\hline Configuracional & $433^{*}$ & 340 & .10 & .95 & .94 & $.03[.02, .03]$ \\
\hline Escalar & $433^{*}$ & 351 & .10 & .95 & .94 & $.03[.02, .03]$ \\
\hline
\end{tabular}

Nota. chi cuadrado $\left(\chi^{2}\right)$; grados de libertad (gl); standardized root mean square residual (SRMR); comparative fit index (CFI), TurkerLewis index (TLI), root mean square error of approximation (RMSEA).

${ }^{a}$ La Invarianza métrica no converge con el estimador WLSM para datos categóricos binarios.

${ }^{\mathrm{b}}$ De acuerdo con la Organización Mundial de la Salud [OMS] (2014) la adolescencia abarca desde los 10 a los 19 años, y se ubica antes de la edad adulta.

$* p<.05$.

\section{Validez convergente y discriminante}

Con respecto a la validez convergente, la BHS de 20 reactivos presentó una correlación positiva y estadísticamente significativa con el BDI $(r=.44, p<$ $.001)$ y el BAI $(r=.39, p<.001)$. En términos de validez predictiva, la BHS explicó el $20 \%$ de la proporción de la varianza de depresión $\left(F=188 ; p<.01 ; R^{2}=.20\right)$ y el $15 \%$ de la varianza de ansiedad $(F=134 ; p<.01$; $\left.R^{2}=.15\right)$.
Además, en términos de validez discriminante, se contrastaron los resultados de la BHS con los resultados de la BAI y el BDI para determinar la precisión de la BHS para discriminar casos con síntomas ansiedad y con depresión. Por tanto, se analizó la curva ROC (receiver operating characteristic) y resultó un área bajo la curva de $.87 \%$ (IC 95\% [.79, .95]). Asimismo, se obtuvo que el punto de corte de $\geq 4$ discriminó entre estudiantes con y sin síntomas ansiedad con una sensibilidad del $1.00 \%$ y una especificidad del .72. Con respecto al con- 
traste con síntomas de depresión, resultó un área bajo la curva ROC de $.90 \%$ (IC 95\% [.80, .99]; con un punto de corte de $\geq 4$ que logró discriminar entre estudiantes con y sin síntomas de depresión con una sensibilidad de .75 y una especificidad del .79

\section{Fiabilidad}

El coeficiente omega de McDonald ( $\omega$ ) para la solución final de la BHS de 20 reactivos fue de .89 (Sentimientos sobre al Futuro, $\omega=.73$; Falta de Motivación, $\omega$ $=.64$; y Expectativas sobre el Futuro, $\omega=.50$ ). Los análisis de fiabilidad de la BHS por grupos arrojaron un $\omega$ de .87 para el grupo de los hombres y .90 para las mujeres; para los adolescentes y adultos fue de .89. Asimismo, el valor del coeficiente omega para los estudiantes del nivel medio superior fue de $.90 \mathrm{y}$ de .88 para los estudiantes de nivel superior.

\section{Discusión}

El presente estudio tuvo como objetivo analizar la estructura factorial interna y las propiedades psicométricas de la BHS en estudiantes de educación media y superior en contextos de pobreza de México. Constatamos que la BHS es válida y fiable para identificar a los estudiantes de educación media superior y superior en contextos de pobreza en México con desesperanza de vida. Es el segundo estudio en el mundo que demuestra empíricamente que la desesperanza surge como un factor latente de segundo orden con tres factores primarios (Sentimientos sobre el Futuro, Falta de Motivación y Expectativas sobre el Futuro) como lo propuso teóricamente Beck et al. (1974), y el primero que arroja una estructura factorial de tres factores correlacionados igual a la escala original. También, los datos de la investigación muestran un ajuste al modelo de Beck et al. (1974) de dos factores correlacionados: un factor con reactivos negativos y el otro con reactivos positivos. Además, en el presente estudio se aceptan los veinte reactivos propuestos originalmente. Por lo tanto, se cuenta con un instrumento culturalmente válido para identificar la desesperanza de vida que permita prevenir el desarrollo de conductas de depresión y suicidio, y de esta manera intervenir la dinámica epidemiológica de dichas psicopatologías en México.

La BHS es fiable para poblaciones clínicas y no clínicas. De acuerdo con el estudio la escala arrojó una alta fiabilidad $(\omega=.89)$. Los resultados del presente estudio coincidieron con lo reportado por Beck et al. $(1974 ; \alpha=.93)$ y Zhang et al. (2015; $\alpha=.95)$ quienes trabajaron con pacientes con intento suicida, y con Chang et al. (1994; $\omega=.95)$, Steed $(2001 ; \alpha=.88$, Aloba et al. $(2018 ; \alpha=.87)$, Hanna et al. (2011; R-K = .87) y Aloba et al. (2016) $(\alpha=$ .87) con universitarios y con Szabó et al. (2016) en población clínica psiquiátrica $(\alpha=.89)$. De este modo, se observó que los resultados también coincidían con lo reportado por Aloba et al. (2015) en población clínica psiquiátrica $(\alpha=.92)$, y con Zhang et al. $(2015 ; \alpha=.90)$, Satorres et al. $(2016 ; \alpha=.93)$ y Kao et al. $(2012 ; \alpha=.85)$, en población general. No obstante, los resultados del presente estudio difieren de los publicados por Gonzales-Huerta et al. (2013) con población clínica no psiquiátrica $(\alpha=.73)$, Aloba et al. (2016) en cuidadores familiares de pacientes psiquiátricos $(\alpha=.72)$, Kocalevent et al. (2016) en población general $(\alpha=.81)$, Pompili et al. $(2007 ; \alpha=.73)$ y González $(2009 ; \alpha=.83)$ en universitarios. De acuerdo con los análisis de este estudio, la BHS es fiable para los estudiantes mexicanos en contextos de pobreza, Asimismo, las diferencias encontradas con los demás estudios son esperadas toda vez que se utilizaron distintas pruebas de fiabilidad y poblaciones de estudios

La estructura factorial de la BHS parece ser diferente para cada población. Se demostró una estructura factorial de la BHS en los estudiantes en contextos de pobreza en México igual a la propuesta original por Beck et al. (1974) con los 20 reactivos originales. Sin embargo, el presente estudio difiere con la solución final que propusieron Beck et al. al eliminar los reactivos cinco y diez. Asimismo, los análisis factoriales confirmatorios demostraron que el presente estudio difiere con lo obtenido por Boduszek y Dhingra (2016), Aloba et al. (2018) y González (2009) con estudiantes universitarios. Por otro lado, los datos de la presente investigación demostraron coincidir con los modelos y soluciones finales sugeridos por Hanna et al. (2011), Chang et al. (1994), Steed et al. (1994) y Pompilini (2007) con universitarios. Contrariamente, los resultados fueron diferentes con los datos obtenidos con población clínica en Taiwán (Kao et al., 2012), Colombia (Rueda-Jaimes et al., 2016), Alemania (Spangenberg et al., 2016) y África (Kagotho et al., 2018).

Por lo tanto, las coincidencias y diferencias en la estructura factorial parecen estribar en el método de análisis y estimación empleados. En este sentido, los estudios que coincidieron con Beck et al. (1974) se caracterizaron por usar el análisis factorial con métodos de estimación robusta; a excepción del estudio presentado por Iliceto y Fino (2015), quienes cambiaron la escala de respuesta dicotómica a tipo Likert de cinco puntos, lo que permitió tener datos con distribución más normal y proceder con el análisis factorial con el método de estimación de máxima verosimilitud. Por otro lado, los estudios que no presentaron una similitud con la estructura original de la 
BHS coincidieron en utilizar métodos de análisis no apropiados para datos categóricos dicotómicos.

La BHS tiene validez concurrente, predictiva y discriminante en los estudiantes de educación media y superior en contextos de pobreza en México. El análisis de la BHS arrojó correlaciones positivas y significativas con las variables de depresión y ansiedad. Los resultados de este estudio coincidieron con lo reportado por Szabó et al. (2016), Zhang et al. (2015), Aloba et al. (2015), Kao et al. (2012), Spangenberg et al. (2016), Kocalevent et al. (2016), Aloba et al. (2016), Iliceto y Fino (2015), Kliem et al. (2018), Aloba et al. (2018), Hanna et al. (2011) y González (2009). Además, se demostró la validez predictiva de la BHS, dado que la desesperanza de vida predice la depresión y la ansiedad. En relación con la validez discriminante, se ha indicado que un punto de corte de $\geq 4$ en la BHS puede ser útil para detectar a estudiantes con y sin síntomas de ansiedad y depresión.

Las fortalezas del estudio son el tamaño de la muestra, las pruebas utilizadas para analizar los datos, y los resultados son generalizables para todos los estudiantes de educación media superior y superior en contextos de pobreza de México. El tamaño de la muestra permitió evitar el error tipo I y lograr estabilidad en el análisis factorial. Además, las pruebas utilizadas fueron las idóneas para datos categóricos no normales, por lo que el uso de modelos robustos permitió mayor precisión en los análisis y resultados. Por otro lado, la debilidad del estudio fue que solo participaron estudiantes de educación media y superior. Por lo tanto, se recomienda que las siguientes investigaciones incluya población en general, y se sugiere aplicar el instrumento en espacios públicos como plazas comerciales, mercados, centros de recreación y esparcimiento, parques, entre otros.

En conclusión, se demostró que la BHS aplicada en estudiantes de educación media y superior en un contexto de pobreza en México es fiable y válida para identificar a los estudiantes (adolescentes y adultos) con desesperanza de vida, en un contexto de pobreza en México. La identificación oportuna de la desesperanza de vida permitirá, en conjunto con profesionales y población en general, elaborar planes, estrategias, programas y acciones para prevenir la depresión y el suicidio en México.

\section{Conflicto de intereses}

Los autores declaran no tener conflicto de intereses.

\section{Referencias}

Aguilar, E., Hidalgo, M., Cano, R., López, J., Campillo, M., y Hernández, J. (1995). Estudio prospectivo de la desesperanza en pacientes psicóticos de inicio: características psicométricas de la escala de desesperanza de Beck en este grupo. Anales de Psiquiatría, 11(4), 121-125.

Aloba, O., Ajao, O., Alimi, T., \& Esan, O. (2016). Psychometric properties and correlates of the beck hopelessness scale in family caregivers of Nigerian patients with psychiatric disorders in Southwestern Nigeria. Journal of Neurosciences in Rural Practice, 7(5), 18-25. doi:10.4103/0976-3147.196434

Aloba, O., Akinsulore, A., Mapayi, B., Oloniniyi, I., Mosaku, K., Alimi, T., \& Esan, O. (2015). The Yoruba version of the Beck Hopelessness Scale: Psychometric characteristics and correlates of hopelessness in a sample of Nigerian psychiatric outpatients. Comprehensive Psychiatry, 56, 258-271. doi:10.1016/j.comppsych.2014.09.024

Aloba, O., Awe, O., Adelola, A., Olatunji, P., \& Aloba, T. (2018). Psychometric adaptation of the Beck Hopelessness Scale as a self-rated suicide risk screening instrument among Nigerian university students. Journal of the American Psychiatric Nurses Association, 24(5), 433-443. doi:10.1177/1078390318762054

American Psychological Association [APA]. (2017). Ethical principles of psychologists and code of conduct. Recuperado de https://www.apa.org/ethics/code/index

Beck, A. T., Epstein, N., Brown, G., \& Steer, R. A. (1988). An inventory for measuring clinical anxiety: Psychometric properties. Journal of Consulting and Clinical Psychology, 56(6), 893-897. doi:10.1037/0022-006X.56.6.893

Beck, A. T., Ward, C., Mendelson, M., Mock, J., \& Erbaugh, J. (1961). Beck depression inventory (BDI). Arch Gen Psychiatry, 4(6), 561-571.

Beck, A. T., Weissman, A., Lester, D., \& Trexler, L. (1974). The measurement of pessimism: The Hopelessness Scale. Journal of Consulting and Clinical Psychology, 42(6), 861-865. doi: $10.1037 / \mathrm{h} 0037562$

Beltrán, M. C., Freyre, M.A., y Hernández-Guzmán, L. (2012). El Inventario de Depresión de Beck: Su validez en población adolescente. Terapia Psicológica, 30(1), 5-13. doi. org/10.4067/S0718-48082012000100001

Bentler, P. M., \& Bonett, D. G. (1980). Significance tests and goodness of fit in the analysis of covariance structures. Psychological Bulletin, 88(3), 588-606. doi:10.1037/00332909.88.3.588

Boduszek, D., \& Dhingra, K. (2016). Construct validity of the beck hopelessness scale (BHS) among university students: A multitrait-multimethod approach. Psychological assessment, 28(10), 1325-1330. doi:10.1037/pas0000245

Burr, E. M., Rahm-Knigge, R. L., \& Conner, B. T. (2018). The differentiating role of state and trait hopelessness in suicidal ideation and suicide attempt. Archives of Suicide Research, 22(3), 510-517. doi:10.1080/13811118.2017.1366960

Chang, E. C., D’Zurilla, T. J., \& Maydeu, A. J. C. T. (1994). Assessing the dimensionality of optimism and pessimism using a multimeasure approach. Cognitive Therapy and Research, 18(2), 143-160. doi:10.1007/bf02357221

Consejo Nacional de Evaluación de Política de Desarrollo Social [CONEVAL] (2016). Medición de la pobreza, Estados Unidos Mexicanos, 2010-2015 (Archivo de datos y libro de códigos). Recuperado de http://www.coneval.org.mx/Medicion/Paginas/ Pobreza-municipal.aspx. 
Córdova, M., y Rosales, J. C. (2011). Consistencia interna y estructura factorial de la Escala de Desesperanza de Beck en estudiantes mexicanos. Revista de Psicología (PUCP), 29(2), 289-309. doi:10.1080/17542863.2017.1417457

Dyce, J. A. (1996). Factor structure of the Beck Hopelessness Scale. Journal of Clinical Psychology, 52(5), 555-558. doi:10.1002/(SICI)1097-4679(199609)52:5<555::AIDJCLP10>3.0.CO;2-D

Flora, D. B., \& Curran, P. J. (2004). An empirical evaluation of alternative methods of estimation for confirmatory factor analysis with ordinal data. Psychological Methods, 9, 466491. doi:10.1037/1082- 989X.9.4.466

Gabaldón, N. (1980). Algunos conceptos de muestreo. Caracas, Venezuela: División de Publicaciones. Facultad de Ciencias Económicas y Sociales. Universidad Central de Venezuela.

Gonzales-Huerta, L., Contreras-Pulache, H., Mori-Quispe, E., Hinostroza-Camposano, W., Pérez-Campos, P., Black, C., y Lam-Figueroa, N. (2013). Escala de desesperanza de Beck: evaluación de la consistencia interna y la estructura factorial para una población de puérperas adolescentes del Instituto Nacional Materno Perinatal. Enero a mayo 2010. Revista Peruana de Epidemiología, 17(2), 1-8.

González, C. (2009). Propiedades psicométricas de la escala de desesperanza de Beck en una muestra bogotana. Psychologia. Avances de la disciplina, 3(2), 17-30.

Hanna, D., White, R., Lyons, K., McParland, M. J., Shannon, C., \& Mulholland, C. (2011). The structure of the Beck Hopelessness Scale: A confirmatory factor analysis in UK students. Personality and Individual Differences, 51(1), 1722. doi:10.1016/j.paid.2011.03.001

Horwitz, A. G., Berona, J., Czyz, E. K., Yeguez, C. E., \& King, C. A. (2017). Positive and negative expectations of hopelessness as longitudinal predictors of depression, suicidal ideation, and suicidal behavior in high-risk adolescents. Suicide and LifeThreatening Behavior, 47(2), 168-176. doi:10.1111/sltb.12273

Hu, L. T., \& Bentler, P. M. (1999). Cutoff criteria for fit indexes in covariance structure analysis: Conventional criteria versus new alternatives. Structural Equation Modeling: A Multidisciplinary Journal, 6(1), 1-55. doi:10.1080/10705519909540118

Huang, P.-H. (2017). Asymptotics of AIC, BIC, and RMSEA for Model Selection in Structural Equation Modeling. Psychometrika, 82(2), 407-426. doi:10.1007/s11336-0179572-y

Iliceto, P., \& Fino, E. (2015). Beck Hopelessness Scale (BHS). European Journal of Psychological Assessment, 31(1), 31-37. doi: 0.1027/1015-5759/a000201

Innamorati, M., Lester, D., Balsamo, M., Erbuto, D., Ricci, F., Amore, M., \& Pompili, M. (2014). Factor validity of the Beck Hopelessness Scale in Italian medical patients. Journal of Psychopathology and Behavioral Assessment, 36(2), 300-307. doi.org/10.1007/s10862-013-9380-3

Instituto Nacional de Estadística y Geografía [INEGI] (2017a). Estadísticas de mortalidad: 1994-2017 (Archivo de datos y libro de códigos). Recuperado de https://www.inegi.org.mx/ temas/mortalidad/

Instituto Nacional de Estadística y Geografía [INEGI] (2017b). Encuesta nacional de Hogares (ENH). Tabulados básicos 2018 (Archivo de datos y libro de códigos). Recuperado de http://www.beta.inegi.org.mx/programas/enh/2017/
Jurado, S., Villegas, M. E., Méndez, L., Rodríguez, F., Loperena, V., y Varela, R. (3 de junio de 1998). La estandarización del Inventario de Depresión de Beck para los residentes de la Ciudad de México. Salud Mental, 21, 26-31. Recuperado de http://revistasaludmental.mx/index.php/salud_mental/article/ viewFile/706/705

Kagotho, N., Bowen, N. K., Ssewamala, F. M., Vaughn, M. G., \& Kirkbride, G. (2018). Cross-national validity of the Beck Hopelessness Scale for children and adolescents: findings from the youth save-impact study Kenya. International Journal of Culture and Mental Health, 1-13. doi:10.1080/175 42863.2017.1417457

Kao, Y.-C., Liu, Y.-P., \& Lu, C.-W. (2012). Beck hopelessness scale: Exploring its dimensionality in patients with schizophrenia. Psychiatric Quarterly, 83(2), 241-255. doi: 10.1007/s11126-011-9196-9

Kliem, S., Lohmann, A., Mößle, T., \& Brähler, E. (2018). Psychometric properties and measurement invariance of the Beck hopelessness scale (BHS): Results from a German representative population sample. BMC Psychiatry, 18(110), 2-11. doi:10.1186/s12888-018-1646-6

Kocalevent, R. D., Finck, C., Pérez-Trujillo, M., Sautier, L., Zill, J., \& Hinz, A. (2016). Standardization of the Beck Hopelessness Scale in the general population. Journal of Mental Health, 1-7. doi:10.1080/09638237.2016.1244717

Koran, J. (2016). Preliminary proactive sample size determination for confirmatory factor analysis models. Measurement and Evaluation in Counseling and Development, 49(4), 296-308. doi:10.1177/0748175616664012

Lane, R., \& Miranda, R. (2018). The effects of familial acculturative stress and hopelessness on suicidal ideation by immigration status among college students. Journal of American College Health, 66(2), 76-86. doi:10.1080/0744848 1.2017.1376673

Liang, X., \& Yang, Y. (2014). An evaluation of WLSMV and Bayesian methods for confirmatory factor analysis with categorical indicators. International Journal of Quantitative Research in Education, 2, 17-38. doi:10.1504/IJQRE.2014.060972

McDonald, R. P. (2014). Factor analysis and related methods. New York: Psychology Press.

Morata-Ramírez, M. Á., Holgado Tello, F. P., Barbero-García, M. I., y Mendez, G. (2015). Análisis factorial confirmatorio. Recomendaciones sobre mínimos cuadrados no ponderados en función del error Tipo I de Ji-cuadrado y RMSEA. Acción Psicológica, 12(1), 79. doi:10.5944/ap.12.1.14362

Nissim, R., Flora, D. B., Cribbie, R. A., Zimmermann, C., Gagliese, L., \& Rodin, G. (2010). Factor structure of the Beck Hopelessness Scale in individuals with advanced cancer. Psycho-Oncology, 19(3), 255-263. doi: 10.1002/pon.1540

Organización Mundial de la Salud [OMS]. (2014). Salud para los adolescentes del mundo: Una segunda oportunidad en la segunda década. Geneva: Autor Recuperado de

Organización Mundial de la Salud [OMS] (2017). Depression and Other Common Mental Disorders. Global Healt Estimates. (OMS CC BY-NC-SA3.0 IGO.). Geneva: Autor. Recuperado de http://apps.who.int/iris/bitstream/handle/10665/254610/ WHO-MSD-MER-2017.2-eng.pdf.

Pérez, S., Marco, J. H., \& García-Alandete, J. (2017). The role of hopelessness and meaning in life in a clinical sample with 
non-suicidal self-injury and suicide attempts. Psicothema, 29(3), 323-328. doi:10.7334/psicothema2016.2

Pompili, M., Tatarelli, R., Rogers, J. R., \& Lester, D. (2007). The Hopelessness Scale: A Factor Analysis. Psychological Reports, 100(2), 375-378. doi:10.2466/pr0.100.2.375-378

Reglamento de la ley general de salud en materia de investigación para la salud, 6 de enero de 1987. Cámara de Diputados México (1987) (Legislado). Recuperado de http://www. ordenjuridico.gob.mx/Documentos/Federal/html/wo88535. html

Rueda-Jaimes, G. E., Castro-Rueda, V. A., Rangel-MartínezVillalba, A. M., Moreno-Quijano, C., Martinez-Salazar, G. A., $\&$ Camacho, P. A. (2016). Validation of the Beck Hopelessness Scale in patients with suicide risk. Revista de Psiquiatría y Salud Mental, 11(2), 86-93. doi:10.1016/j.rpsm.2016.09.004

Sánchez-Teruel, D., Muela-Martínez, J. A., y García-León, A. (2018). Variables de riesgo y protección relacionadas con la tentativa de suicidio. Revista de Psicopatología y Psicología Clínica, 23(3), 221-229. doi: https://doi.org/10.5944/rppc. vol.23.num.3.2018.19106

Satorres, E., Ros, L., Meléndez, J. C., Serrano, J. P., Latorre, J. M., $\&$ Sales, A. (2016). Measuring elderly people's quality of life through the Beck Hopelessness Scale: a study with a Spanish sample. Aging and Mental Health, 22 (2), 239-244. doi:10.10 80/13607863.2016.1247427

Secretaría de Educación Jalisco (2017). Estadística educativa por región 2017: ciclo escolar 2017-2018 (Archivo de datos y libro de códigos. Recuperado de http://indicadores.sej.gob. $\mathrm{mx} /$ publicaciones/Ini2017-2018/index.htm

Silva, D., Valdivia, M., Vicente, B., Arévalo, E., Dapelo, R., y Soto, C. (2017). Intento de suicidio y factores de riesgo en una muestra de adolescentes escolarizados de Chile. Revista de Psicopatología y Psicología Clínica, 22(1), 33-42. doi: https:// doi.org/10.5944/rppc.vol.22.num.1.2017.16170

Sociedad Mexicana de Psicología [SMP]. (2010). Código ético del psicólogo. México: Trillas.

Spangenberg, L., Zenger, M., Garcia-Torres, F., Mueller, V., Reck, M., Mehnert, A., \& Vehling, S. (2016). Dimensionality, stability, and validity of the Beck Hopelessness Scale in cancer patients receiving curative and palliative treatment. Journal of Pain and Symptom Management, 51(3), 615-622 doi: 10.1016/j.jpainsymman.2015.11.008

Steed, L. (2001). Further Validity and Reliability Evidence for Beck Hopelessness Scale Scores in a Nonclinical Sample. Educational and Psychological Measurement, 61(2), 303316. doi:10.1177/00131640121971121

Steer, R. A., Beck, A. T., \& Brown, G. K. (1997). Factors of the Beck Hopelessness Scale: Fact or artifact? Multivariate Experimental Research, 11, 131-144.

Steer, R. A., Iguchi, M. Y., \& Platt, J. J. (1994). Hopelessness in IV drug users not in treatment and seeking HIV testing and counselling. Drug and Alcohol Dependence, 34(2), 99-103. doi:https://doi.org/10.1016/0376-8716(94)90129-5

Szabó, M., Mészáros, V., Sallay, J., Ajtay, G., Boross, V., UdvardyMészáros, À., \& Perczel-Forintos, D. (2016). The beck hopelessness scale; specific factors of method effects. European Journal of Psychological Assessment, 32(2), 111118. doi:10.1027/1015-5759/a000240

Vázquez, O. G., Castillo, E. R., García, A. M., Ponce, J. L. A., Avitia, M. Á. Á., y Aguilar, S. A. (2015). Propiedades Psicométricas del Inventario de Ansiedad de Beck (BAI) en pacientes con cáncer. Psicooncología, 12(1), 51-58. doi: 10.5209/rev_PSIC.2015.v12.n1.48903

World Medical Association [WMA] (2017). WMA Declaration of Helsinki - Ethical Principles for Medical Research Involving Human Subjects. Recuperado de https://www.wma.net/ policies-post/wma-declaration-of-helsinki-ethical-principlesfor-medical-research-involving-human-subjects/

Young, M. A., Halper, I. S., Clark, D. C., Scheftner, W., \& Fawcett, J. (1992). An item-response theory evaluation of the Beck Hopelessness Scale. Cognitive Therapy and Research, 16(5), 579-587. doi:10.1007/BF01175143

Zhang, W. C., Jia, C. X., Hu, X., Qiu, H. M., \& Liu, X. C. (2015). Beck Hopelessness Scale: Psychometric properties among rural Chinese suicide attempters and on-Attempters. Death Studies, 39(7), 442-446.n doi:10.1080/07481187.2014.970300 
Anexo I

Escala de Desesperanza (Hopelessness Scale, BHS)

Instrucciones: Señale si las siguientes afirmaciones se ajustan o no a su situación personal. Las opciones de respuestas son: verdadera (V) o falsa (F).

\begin{tabular}{|c|c|c|c|}
\hline Núm. & Reactivo & $\mathrm{V}$ & $\mathrm{F}$ \\
\hline 1 & Espero el futuro con esperanza y entusiasmo. & $($ ) & ( ) \\
\hline 2 & Puedo darme por vencido, renunciar, ya que no puedo hacer mejor las cosas por mí mismo. & $($ ) & $($ ) \\
\hline 3 & Cuando las cosas van mal me alivia saber que las cosas no pueden permanecer tiempo así. & $($ ) & $($ ) \\
\hline 4 & No puedo imaginar cómo será mi vida dentro de 10 años. & $($ ) & $($ ) \\
\hline 5 & Tengo bastante tiempo para llevar a cabo las cosas que quisiera poder hacer. & $($ ) & $($ ) \\
\hline 6 & En el futuro, espero conseguir lo que me pueda interesar. & $($ ) & $($ ) \\
\hline 7 & Mi futuro me parece obscuro. & $($ ) & $($ ) \\
\hline 8 & Espero más cosas buenas de la vida que lo que la gente suele conseguir por término medio. & ( ) & ( ) \\
\hline 9 & No logro hacer que las cosas cambien, y no existen razones para creer que pueda en el futuro. & $($ ) & ( ) \\
\hline 10 & Mis pasadas experiencias me han preparado bien para mi futuro. & $($ ) & $($ ) \\
\hline 11 & Todo lo que puedo ver delante de mí es más desagradable que agradable. & $($ ) & $($ ) \\
\hline 12 & No espero conseguir lo que realmente deseo. & $($ ) & $($ ) \\
\hline 13 & Cuando miro hacia el futuro, espero que seré más feliz de lo que soy ahora. & $($ ) & $($ ) \\
\hline 14 & Las cosas no marchan como yo quisiera. & $($ ) & $($ ) \\
\hline 15 & Tengo una gran confianza en el futuro. & $($ ) & $($ ) \\
\hline 16 & Nunca consigo lo que deseo, por lo que es absurdo desear cualquier cosa. & $($ ) & $($ ) \\
\hline 17 & Es muy improbable que pueda lograr una satisfacción real en el futuro. & $($ ) & $($ ) \\
\hline 18 & El futuro me parece vago e incierto. & $($ ) & $($ ) \\
\hline 19 & Espero más bien épocas buenas que malas. & $($ ) & $($ ) \\
\hline 20 & No merece la pena que intente conseguir algo que desee, porque probablemente no lo lograré. & $($ ) & $($ ) \\
\hline
\end{tabular}

